\title{
Empirische Erkundungen zum Coaching
}

\section{Astrid Schreyögg}

In der Literatur finden sich immer wieder Klagen, dass im Bereich Coaching noch allzu wenig Empirisches vorliegt. Das ist richtig, denn Coaching hat sich letztlich in der Praxis entwickelt und blieb bis heute primär ein Kind der Praxis. Und welcher Praktiker unterzieht sich denn schon der Mühe, empirische Arbeiten anzufertigen, wenn solche „Hobbyforschung“ dem strengen Blick von Wissenschaftlern selten standhalten kann. Nichtsdestoweniger spricht es sich auch bei eingefleischten Praktikern herum, dass Coaching auf dem Weg seiner Professionsentwicklung einigermaßen gültige Nachweise seiner Wirksamkeit erbringen muss. Das ist schon deshalb wichtig, um den hohen finanziellen Aufwand, der im Allgemeinen mit Coaching verbunden ist, zu rechtfertigen. So hat die empirische Forschung auch hier eine durchaus ernst zu nehmende Legitimationsfunktion.

Spätestens seit der Jahrtausendwende interessierten sich allerdings immer mehr Wissenschaftler für dieses Beratungsformat. Der Australier Grant (2006) stellte für den englischsprachigen Bereich fest, dass zwischen 2001 bis 2005 die Publikationen über Coaching im akademischen Bereich um 266\% angewachsen sind gegenüber dem Zeitraum von 1996 bis 2000. So finden wir heute auch an deutschen Hochschulen und Fachhochschulen immer mehr Forschungsarbeiten zu diesem Bereich. Zwar wirken die Fragestellungen oft etwas zu eng oder zu artifiziell, neuerdings lassen sich aber zunehmend gelungene Kooperationen zwischen Forschung und Praxis beobachten. Das liegt zum einen sicher daran, dass heute immer mehr Forscher/innen Coaching-Ausbildungen absolvieren. Das ergibt sich aber wahrscheinlich auch aus dem Umstand, dass immer mehr Praktiker/innen an Lehrstühlen für Psychologie, Pädagogik, Soziologie oder Betriebswirtschaftslehre über Coaching promovieren. Auch in der jährlich tagenden Academy of Management, der größten US-amerikanischen Konferenz für die Managementforschung, treten hier und da Referenten mit Coachingthemen auf. Zwar wächst seit einigen Jahren auch das Interesse der Professionssoziologie am Coaching (Kühl 2008), die daraus resultierenden Arbeiten sind aber meistens eher der Konzeptentwicklung und weniger der Empirie geschuldet. Das vorliegende Heft soll nun einen Beitrag zur Abhilfe dieses vermeintlichen Defizits von empirischen Arbeiten zum Coaching leisten.

Online publiziert: 14.01 .2014

(C) Springer Fachmedien Wiesbaden 2014

Dr. A. Schreyögg $(\bowtie)$

Breisgauer Str. 29, 14129 Berlin, Deutschland

E-Mail: info@schreyoegg.de 
Im ersten Beitrag präsentieren Doris Cornils, Anna Mucha und Daniela Rastetter ein Projekt, bei dem sie Frauen qua Coaching trainiert haben, mikropolitische Prozesse in Organisationen besser zu durchschauen und zu handhaben. Die Autorinnen entwickelten im Rahmen eines Forschungsprojekts zunächst das „Mikropolitische Kompetenzmodell“ und belegten anschließend anhand von Fallbeispielen seinen Nutzen für weibliche Führungskräfte. Doris Klenner und Barbara Bischofberger untersuchten die Übungsformen von Coaching-Ausbildungen. Sie ermittelten im Rahmen einer Online-Befragung von 209 Coaching-Trainern eine Vielzahl von Daten über deren Vorberuf, Dauer der Coaching-Tätigkeit usw. Das Fazit der Autorinnen ist, dass in den meisten Ausbildungen Triaden- bzw. Kleingruppenarbeit einen großen Raum einnimmt. Daraus ergibt sich allerdings die Forderung, dass die Spezifika dieser Arbeitsform doch etwas genauer untersucht werden sollten. Wolfgang Kühl zeigt anhand von 18 Interviews mit Führungskräften aus der Sozialen Arbeit, wie diese durch Coaching besonders ihre Fähigkeit zur Konfliktbewältigung verbessern konnten.

Daran anschließend präsentieren Carsten Schermuly und Franziska Bohnhardt eine Befragung von 20 erfahrenen Coaches über negative Effekte von Coaching bei Coaches und bei Klient/innen. Die Autoren kommen zu ähnlichen Ergebnissen wie kürzlich von Brigitte Schigl (2013) in OSC für die Supervision beschrieben. Auch diese Autoren kommen vorrangig zu dem Ergebnis, dass Meta-Consulting, d. h. Supervision der Coaches, dringend erforderlich ist, um Gefahren zu vermeiden. Doris Dietachmair und Arthur Drexler haben die Wirkungen eines spezifischen Planspiels, das in die Coachings von Arbeitssuchenden integriert war, untersucht. Sie können zeigen, dass die Klienten durch dieses Planspiel bedeutend mehr Selbstvertrauen gewinnen konnten. Zwar spielt professionelle Gesprächsführung im Coaching schon immer eine Rolle, Michael Cramer und Anne Sauer haben sie aber durch eine besondere Variante, das „Motivational Interviewing“, erheblich erweitert. Die Autoren betonen, dass Coaching durch diese Form der Gesprächsführung noch effizienter wird.

Volker Jörn Walpuski hat sich mit einem zunehmend bedeutsamen Thema beschäftigt: mit Regelungen für die computergestützte Kommunikation in Unternehmen. Er fragt: „Soll denn heute jeder rund um die Uhr zugänglich sein?“ und hat diese Zumutung bei zahlreichen Firmen untersucht. Er meint, dass die Reflexion der Techniknutzung auch im Coaching eine Rolle spielen sollte. In einem weiteren Diskurs-Beitrag rollt Manfred Helbing ,die Bedeutung der Beziehung im Coaching“ facettenreich auf. Seinen Überlegungen können wir nur zustimmen.

\section{Literatur}

Grant, A. M. (2006). Workplace and executive coaching: A bibliography from the scholarly business literature. In R. Stober \& A. M. Grant (Hrsg.), Evidence based coaching handbook. Putting best practices to work for your clients (S. 367-388). Hoboken: Wiley.

Kühl, S. (2008). Coaching und Supervision. Zur personenorientierten Beratung in Organisationen. Wiesbaden: Springer-VS.

Schigl, B. (2013). Wie gefährlich kann Supervision sein? OSC, 20(1), 35-51. 